\title{
Use of hospital inpatient care in adolescence
}

\author{
Jane Henderson, Michael Goldacre, David Yeates
}

\begin{abstract}
Epidemiological information about detailed patterns of physical morbidity within the adolescent age group is not generally available. To illustrate the distinctive patterns of morbidity indicated by the use of hospital inpatient care, hospital admission rates in the Oxford region (1979-86) were analysed at each single year of age from 10 to 19 years. At the age of 10 years $22 \%$ of general hospital admissions were to paediatrics, $24 \%$ to general surgery, $23 \%$ to ear, nose, and throat surgery, and $20 \%$ to trauma and orthopaedics. By 14 years of age only $6 \%$ of general hospital admissions were to paediatrics. By 16 years of age $24 \%$ of general hospital admissions of young women were to gynaecology and $40 \%$ of admissions of young men were to trauma and orthopaedics. The most common reason for hospital admission in young men was head injury and the second most common was appendicectomy. Termination of pregnancy was the single most common reason for admission for girls aged 15 and 16 years; childbirth and terminations were the most common reasons for admission in girls aged 17-19 years and over. Self poisoning was also common in older teenage girls. Younger girls were admitted most commonly for tonsillectomy. Most admissions of adolescents are thus for surgical rather than medical reasons and some of the most common individual reasons for admission are attributable to behavioural factors rather than disease processes.

(Arch Dis Child 1993; 69: 559-563)
\end{abstract}

Adolescence is a time not only of rapid physical development, but also of considerable change in patterns of morbidity and use of hospital care. The conventional groupings of vital and health care statistics into five year age groups, and commonly into broader age groups than these, mean that the distinctive features of these changes are not highlighted and have not commonly been studied in detail on a population basis in England. ${ }^{1}$ The transition from childhood to adulthood may also be a difficult age range for placing patients in suitable ward space. ${ }^{2}$

To illustrate the distinctive patterns of morbidity and use of inpatient care in this age group, we report the common reasons for hospital admission at each single year of age between 10 and 19 years.

\section{Methods}

Data from the Oxford record linkage study were used. Six health districts in the Oxford region were covered by full, linked data collection in general hospital specialties from the mid-1970s until the implementation of Körner data sets in April 1987. In this study we used data from 1979 (when the 9th revision of the International Classification of Diseases was implemented) to 1986 inclusive. At this time the six districts included an average of 330000 people aged 10-19 years. Statistical abstracts of all inpatient and day care in general specialties were identified - that is, in all hospital specialties including gynaecology but excluding maternity and psychiatry - in these six districts. Obstetric data were available for two districts and psychiatric data for one district only. For completeness we refer to these briefly but, unless otherwise stated, our findings and calculations refer to the use of general hospital specialties.

Linked records were used to provide person based counts of admissions to hospital for each diagnosis, operation, and specialty in the following ways. For multiple admissions for any one person, each person was counted once only at each single year of age. Thus, for example, a patient admitted several times for asthma at the age of 10 years and several times at the age of 11 counted as one patient admitted at 10 and one at 11 years of age. People with more than one admission for an operation were counted in similar ways. People admitted more than once to a specialty were counted once only in each specialty at each year of age. In the 'general specialties total' people admitted to more than one specialty were counted once only at each year of age to provide data on individuals 'ever admitted' at each age.

Specialties were designated in the analyses as the specialty of the consultant responsible for the patient's care at the time of discharge from hospital. It is possible that some patients discharged by consultants in specialties other than paediatrics were also seen on the wards by paediatricians.

Clinical data in the Oxford record linkage study were coded from 1979-86 according to the ninth revision of the International Classification of Diseases and the third revision of the Office of Population Censuses and Surveys' operation codes. ${ }^{34}$ The main diagnosis and operation at three digits on the record of each admission were selected for the analysis. A small number of similar disorders with different clinical codes at the three digital level were grouped together. These are shown in the tables and include, for example, the five different three digit codes for appendicectomy, the two different diagnostic codes for head injury and concussion (subsequently referred to in the text as head injury), and the eight different codes covering operations on the tonsils and adenoids. 
We used a number of rules of precedence in deciding how to count combinations of diagnoses and operations. For example, an admission with a recorded diagnosis of abdominal pain, and an appendicectomy, was counted as appendicectomy and not as abdominal pain. Thus the admissions for abdominal symptoms, in the tables, are those for abdominal symptoms without appendicectomy. An admission for tonsillectomy was counted as such and not as the diagnosis on the record. Thus the admissions for upper respiratory tract infections, recorded in the tables, exclude patients in whom the diagnosis was recorded at a hospital admission for tonsillectomy. Our use of precedence in the analysis of certain gynaecological disorders was a little more complicated. If a girl had more than one admission within six months for the following, she was counted once only in the following order of precedence: termination of pregnancy, dilatation and curettage, postnatal operations, and disorders of menstruation (without operation). We did this to ensure that successive admissions relating to the same pregnancy, or menstrual disorder, were counted only once. It is possible, however, that by doing this we may have slightly underestimated the counts of operations in these categories which were in fact unrelated.

Results are presented as average annual population based admission rates, separately for males and females, at single years of age. Population figures are not available by individual health district in single years of age. Accordingly, the national figures for single years within the age groups $10-14$ and 15-19 years were applied to the number of people in each five year age group in the six districts to give an estimate of the local number of people in each single year of age.

\section{Results}

SPECIALTIES

Admission rates to all general specialties combined were higher in boys than girls up to the age of 14 years, and higher in girls than boys thereafter (table 1). Admission rates for boys were lowest at ages 15 and 16 years and lowest for girls at ages 11 and 12 years. Table 1 gives detailed profiles of admission rates by age and specialty. For example, for boys aged 10 years $21 \%$ of general hospital admissions were to paediatrics, $28 \%$ to general surgery, $19 \%$ to ear, nose, and throat surgery, and $20 \%$ to trauma and orthopaedics.

For 10 year old girls $22 \%$ of general hospital admissions were to paediatrics, $18 \%$ to general surgery, $27 \%$ to ear, nose, and throat surgery, and $20 \%$ to trauma and orthopaedics. By the age of 14 years, only $6 \%$ of general hospital admissions were to paediatrics in boys compared with $33 \%$ to trauma and orthopaedics and $26 \%$ to general surgery. The corresponding figures for girls were $6 \%$ to paediatrics, $22 \%$ to trauma and orthopaedics, and $21 \%$ to general surgery. By 16 years $24 \%$ of general hospital admissions for girls were to gynaecology and less than $1 \%$ to paediatrics. By 16 years $40 \%$ of general hospital admissions of males were to trauma and orthopaedics. Admission rates for trauma and orthopaedics increased strikingly with age, more so for males than females, across the age range. Admission rates for ear, nose, and throat surgery and ophthalmology decreased with age in males and females.

Admissions for mental illness increased with age in males and females. Admissions for mental handicap peaked at age 14 years. Obstetric admissions increased sharply with age and exceeded admissions to any other specialty for girls from the age of 17 years.

Table 1 Average annual admission rates to each specialty per 10000 people at each age by sex and the range of numbers on which the rates are based

\begin{tabular}{|c|c|c|c|c|c|c|c|c|c|c|c|c|c|}
\hline \multirow[b]{2}{*}{ Specialty } & & \multicolumn{10}{|c|}{ Age (years) } & \multicolumn{2}{|c|}{ Range $^{\star}$} \\
\hline & & 10 & 11 & 12 & 13 & 14 & 15 & 16 & 17 & 18 & 19 & $n_{1}$ & $n_{2}$ \\
\hline $\begin{array}{l}\text { General medicine } \\
\text { General surgery } \\
\text { Paediatrics } \\
\text { Ear, nose, and } \\
\text { throat surgery } \\
\text { Dentistry and } \\
\text { oral surgery } \\
\text { Neurosurgery } \\
\text { Trauma and } \\
\text { orthopaedics } \\
\text { Ophthalmology } \\
\text { Plastic surgery } \\
\text { Other specialties } \\
\text { Gynaecology }\end{array}$ & $\begin{array}{l}\text { M } \\
\mathbf{F} \\
\mathbf{M} \\
\mathbf{F} \\
\mathbf{M} \\
\mathbf{F} \\
\mathbf{M} \\
\mathbf{F} \\
\mathbf{M} \\
\mathbf{F} \\
\mathbf{M} \\
\mathbf{F} \\
\mathbf{M} \\
\mathbf{F} \\
\mathbf{M} \\
\mathbf{F} \\
\mathbf{M} \\
\mathrm{F} \\
\mathbf{M} \\
\mathbf{F} \\
\mathrm{F}\end{array}$ & $\begin{array}{r}2 \cdot 2 \\
1 \cdot 8 \\
129 \cdot 2 \\
59 \cdot 6 \\
97 \cdot 4 \\
74 \cdot 2 \\
88 \cdot 7 \\
90 \cdot 1 \\
15 \cdot 7 \\
15 \cdot 2 \\
2 \cdot 4 \\
0 \cdot 8 \\
95 \cdot 2 \\
66 \cdot 6 \\
9 \cdot 1 \\
8 \cdot 0 \\
9 \cdot 8 \\
7 \cdot 9 \\
15 \cdot 0 \\
9 \cdot 9 \\
2 \cdot 2\end{array}$ & $\begin{array}{r}3 \cdot 2 \\
2 \cdot 4 \\
125 \cdot 5 \\
60 \cdot 4 \\
93 \cdot 4 \\
65 \cdot 1 \\
73 \cdot 4 \\
76 \cdot 2 \\
17 \cdot 3 \\
22 \cdot 8 \\
2 \cdot 6 \\
1 \cdot 4 \\
102 \cdot 3 \\
66 \cdot 3 \\
8 \cdot 8 \\
5 \cdot 7 \\
8 \cdot 7 \\
8 \cdot 4 \\
13.9 \\
11.2 \\
1.7\end{array}$ & $\begin{aligned} 7 \cdot 3 \\
8 \cdot 2 \\
114 \cdot 1 \\
59 \cdot 5 \\
76 \cdot 2 \\
53 \cdot 3 \\
63 \cdot 2 \\
70 \cdot 9 \\
20 \cdot 1 \\
30 \cdot 4 \\
1 \cdot 7 \\
1 \cdot 1 \\
108 \cdot 6 \\
69 \cdot 3 \\
8 \cdot 4 \\
4 \cdot 6 \\
10 \cdot 1 \\
8 \cdot 8 \\
16 \cdot 0 \\
13 \cdot 1 \\
2.6\end{aligned}$ & $\begin{array}{r}16 \cdot 8 \\
18 \cdot 4 \\
102 \cdot 9 \\
66 \cdot 4 \\
48 \cdot 1 \\
36 \cdot 8 \\
58 \cdot 2 \\
66 \cdot 2 \\
21 \cdot 7 \\
36 \cdot 6 \\
2 \cdot 1 \\
1.5 \\
125 \cdot 0 \\
75 \cdot 8 \\
7 \cdot 9 \\
5 \cdot 2 \\
9 \cdot 4 \\
7 \cdot 0 \\
11 \cdot 4 \\
12 \cdot 1 \\
7 \cdot 0\end{array}$ & $\begin{array}{r}28 \cdot 4 \\
41 \cdot 0 \\
106 \cdot 6 \\
79 \cdot 4 \\
25 \cdot 2 \\
22 \cdot 8 \\
62 \cdot 6 \\
62 \cdot 0 \\
24 \cdot 2 \\
35 \cdot 6 \\
2 \cdot 3 \\
0 \cdot 7 \\
137 \cdot 0 \\
84 \cdot 2 \\
7 \cdot 2 \\
4 \cdot 8 \\
11 \cdot 8 \\
7 \cdot 5 \\
12 \cdot 9 \\
14 \cdot 4 \\
26 \cdot 5\end{array}$ & $\begin{aligned} 35 \cdot 5 \\
58 \cdot 4 \\
87 \cdot 6 \\
88 \cdot 3 \\
9 \cdot 8 \\
10 \cdot 7 \\
53 \cdot 9 \\
68 \cdot 6 \\
19 \cdot 8 \\
32 \cdot 6 \\
1 \cdot 3 \\
1 \cdot 4 \\
128 \cdot 1 \\
79 \cdot 6 \\
6 \cdot 9 \\
4 \cdot 7 \\
9 \cdot 9 \\
8 \cdot 4 \\
12 \cdot 9 \\
16 \cdot 6 \\
65 \cdot 7\end{aligned}$ & $\begin{array}{r}38 \cdot 8 \\
64 \cdot 1 \\
81.3 \\
99 \cdot 4 \\
3.6 \\
4.5 \\
47 \cdot 8 \\
58.5 \\
17 \cdot 0 \\
35 \cdot 8 \\
1.7 \\
1.4 \\
145 \cdot 4 \\
84 \cdot 6 \\
4.7 \\
4.0 \\
10 \cdot 2 \\
9 \cdot 1 \\
12.7 \\
15.5 \\
121.3\end{array}$ & $\begin{array}{r}45.8 \\
78.2 \\
91.0 \\
108.1 \\
1.5 \\
1.6 \\
43.9 \\
58.1 \\
18.4 \\
39.8 \\
1.5 \\
1.6 \\
184.3 \\
85.1 \\
5.8 \\
4.1 \\
11.8 \\
8.5 \\
13.1 \\
19.3 \\
158.4\end{array}$ & $\begin{array}{r}55 \cdot 6 \\
82.1 \\
105.5 \\
119.7 \\
0.7 \\
0.6 \\
46.7 \\
57.9 \\
20.3 \\
52.1 \\
2.2 \\
1.1 \\
174.3 \\
84.9 \\
5.0 \\
4.8 \\
13.8 \\
8.5 \\
19.4 \\
20.0 \\
188.1\end{array}$ & $\begin{array}{r}58.9 \\
85.4 \\
103.2 \\
123.0 \\
0.4 \\
0.5 \\
41.6 \\
48.0 \\
22.0 \\
59 \cdot 3 \\
2.2 \\
1.8 \\
174.8 \\
83.1 \\
5.3 \\
3.0 \\
12.8 \\
6.9 \\
11.8 \\
24.7 \\
223.7\end{array}$ & $\begin{array}{r}27 \\
21 \\
1302 \\
693 \\
7 \\
7 \\
668 \\
685 \\
193 \\
177 \\
20 \\
9 \\
1173 \\
774 \\
76 \\
43 \\
111 \\
91 \\
158 \\
107 \\
21\end{array}$ & $\begin{array}{r}946 \\
1218 \\
1701 \\
1755 \\
1200 \\
863 \\
1093 \\
1047 \\
354 \\
846 \\
36 \\
25 \\
2977 \\
1214 \\
113 \\
93 \\
222 \\
129 \\
232 \\
367 \\
3192\end{array}$ \\
\hline $\begin{array}{c}\text { All general } \\
\text { specialties }\end{array}$ & $\begin{array}{l}\mathbf{M} \\
\mathbf{F} \\
\end{array}$ & $\begin{array}{l}460 \cdot 3 \\
335 \cdot 8 \\
\end{array}$ & $\begin{array}{l}447 \cdot 6 \\
319 \cdot 5\end{array}$ & $\begin{array}{l}420 \cdot 1 \\
318 \cdot 8\end{array}$ & $\begin{array}{l}403 \cdot 7 \\
333 \cdot 1\end{array}$ & $\begin{array}{l}418 \cdot 7 \\
379 \cdot 4 \\
\end{array}$ & $\begin{array}{l}365 \cdot 5 \\
435 \cdot 3 \\
\end{array}$ & $\begin{array}{l}363 \cdot 8 \\
498 \cdot 8 \\
\end{array}$ & $\begin{array}{l}417 \cdot 1 \\
563 \cdot 8 \\
\end{array}$ & $\begin{array}{l}437 \cdot 4 \\
620 \cdot 5 \\
\end{array}$ & $\begin{array}{l}435 \cdot 7 \\
660 \cdot 3\end{array}$ & $\begin{array}{l}5511 \\
3860\end{array}$ & $\begin{array}{l}7055 \\
9423\end{array}$ \\
\hline $\begin{array}{l}\text { Mental illnesst } \\
\text { Mental handicapt }\end{array}$ & $\begin{array}{l}\mathbf{M} \\
\mathbf{F} \\
\mathbf{M} \\
\mathbf{F}\end{array}$ & $\begin{array}{r}11 \cdot 1 \\
5 \cdot 2 \\
5 \cdot 9 \\
5 \cdot 2\end{array}$ & $\begin{array}{l}8 \cdot 1 \\
9 \cdot 7 \\
3 \cdot 2 \\
6 \cdot 1\end{array}$ & $\begin{array}{r}13.3 \\
8.7 \\
7.4 \\
8.0\end{array}$ & $\begin{array}{l}12.9 \\
14 \cdot 1 \\
10 \cdot 1 \\
11.8\end{array}$ & $\begin{array}{l}19.0 \\
19.7 \\
12.9 \\
16.4\end{array}$ & $\begin{array}{l}13.4 \\
21.0 \\
10.2 \\
13.5\end{array}$ & $\begin{array}{r}10.1 \\
14.2 \\
9.2 \\
12.9\end{array}$ & $\begin{array}{r}9 \cdot 6 \\
20 \cdot 6 \\
7 \cdot 3 \\
9 \cdot 7\end{array}$ & $\begin{array}{r}16 \cdot 7 \\
20 \cdot 8 \\
7 \cdot 4 \\
8.4\end{array}$ & $\begin{array}{r}19.9 \\
28.7 \\
7.4 \\
6.2\end{array}$ & $\begin{array}{l}32 \\
14 \\
14 \\
14\end{array}$ & $\begin{array}{r}89 \\
106 \\
45 \\
50\end{array}$ \\
\hline $\begin{array}{l}\text { Obstetrics } ¥ \\
\text { Admissions } \\
\text { Deliveries }\end{array}$ & $\begin{array}{l}\mathbf{F} \\
\mathbf{F}\end{array}$ & $\begin{array}{l}0 \\
0\end{array}$ & $\begin{array}{l}0 \\
0\end{array}$ & $\begin{array}{l}0 \\
0\end{array}$ & $\begin{array}{l}0.2 \\
0.2\end{array}$ & $\begin{array}{l}4 \cdot 2 \\
4 \cdot 0\end{array}$ & $\begin{array}{l}21 \cdot 1 \\
19 \cdot 9\end{array}$ & $\begin{array}{l}69 \cdot 9 \\
65 \cdot 3\end{array}$ & $\begin{array}{l}161 \cdot 2 \\
155 \cdot 4\end{array}$ & $\begin{array}{l}257 \cdot 5 \\
246.6\end{array}$ & $\begin{array}{l}379 \cdot 2 \\
360 \cdot 3\end{array}$ & $\begin{array}{l}0 \\
0\end{array}$ & $\begin{array}{l}2510 \\
2385\end{array}$ \\
\hline
\end{tabular}

${ }^{\star} n_{1}$ and $n_{2}$ are the numbers of people in the age group with, respectively, the highest and lowest rates in each specialty.

tRelates to one district only. $¥$ Relates to two districts only. 
Table 2 Hospital admission rates in males expressed as rates per 10000 males at each age, and the range of numbers on which the rates are based, with the top 10 reasons for admission at each age ranked in parentheses

\begin{tabular}{|c|c|c|c|c|c|c|c|c|c|c|c|c|}
\hline \multirow{2}{*}{$\begin{array}{l}\text { Diagnoses (and ICD codes) or } \\
\text { operations (and OPCS } \\
\text { codes in italics) }\end{array}$} & \multicolumn{10}{|l|}{ Age (years) } & \multicolumn{2}{|c|}{ Ranget } \\
\hline & 10 & 11 & 12 & 13 & 14 & 15 & 16 & 17 & 18 & 19 & $n_{1}$ & $n_{2}$ \\
\hline \multicolumn{13}{|l|}{ Upper respiratory tract } \\
\hline infections $(460-5,478)$ & $2 \cdot 7(9)$ & $12 \cdot 8(10)$ & $12 \cdot 5(9)$ & $10 \cdot 0$ & $10 \cdot 0(10)$ & $7 \cdot 5(9)$ & $6 \cdot 7(10)$ & $6 \cdot 8$ & $7 \cdot 4$ & $5 \cdot 9$ & 95 & 165 \\
\hline Asthma (493) & $\cdot 2(8)$ & & $10=)$ & & & & & & & & 67 & 224 \\
\hline Abdominal symptoms (789) & $24 \cdot 4(4)$ & 26 . & 21 . & (4) & & 10 . & $7 \cdot 7(9)$ & $10 \cdot 0(8)$ & $14 \cdot 4(8)$ & $12 \cdot 1(8)$ & 124 & 335 \\
\hline Fractured radius, ulna (813) & $13 \cdot 3$ & & & & & & & & & $3 \cdot 7$ & 60 & 187 \\
\hline 4) & $51.9(1)$ & $50 \cdot 8(1)$ & $53 \cdot 2(1)$ & $54 \cdot 5(1)$ & $52 \cdot 3(1)$ & $46 \cdot 3(1)$ & $51 \cdot 5(1)$ & $67 \cdot 2(1)$ & $61.9(1)$ & $63 \cdot 1(1)$ & 639 & 1085 \\
\hline nds $(870-97)$ & & & & 10 . & & & & & & & 86 & 341 \\
\hline Poisoning $(960-89)$ & $2 \cdot 8$ & & $2 \cdot 6$ & $4 \cdot($ & & $7 \cdot 4(10)$ & $10 \cdot 7(7)$ & $14 \cdot 4(6)$ & $17 \cdot 9(5)$ & $18 \cdot 4(5)$ & 31 & 296 \\
\hline Myringotomy (193) & $22 \cdot 9(6=)$ & $17 \cdot 8(7)$ & $11 \cdot 3(10=)$ & 9 . & 6 . & $5 \cdot 0$ & $3 \cdot 6$ & $2 \cdot($ & $1 \cdot$ & $1 \cdot 0$ & 16 & 282 \\
\hline \multicolumn{13}{|l|}{ Operations on nose } \\
\hline Operations on nasal septum & $12 \cdot 2$ & $14 \cdot 5(9)$ & $16 \cdot 1(5)$ & $17 \cdot 9(3)$ & $22 \cdot 4(3)$ & $22 \cdot 8(3)$ & $19 \cdot 2(3)$ & $20 \cdot 5(3)$ & $22 \cdot 3(3)$ & $19 \cdot 3(4)$ & 150 & 361 \\
\hline $\begin{array}{l}\text { Operations on nasal septum } \\
(212-4)\end{array}$ & 0.6 & & & & & & & $7 \cdot 6$ & $9 \cdot 4(10)$ & $8 \cdot 4(10=)$ & 8 & 135 \\
\hline Tonsils and adenoids $(230-9)$ & $38 \cdot 0(2)$ & $28 \cdot 6(3)$ & (4) & $15 \cdot 5(5)$ & $17 \cdot 1(5)$ & $12 \cdot 6(6)$ & $5(8)$ & $(9)$ & $9 \cdot 8(9)$ & & 130 & 468 \\
\hline Operations on teeth $(250-9)$ & $8 \cdot 3(10)$ & $9 \cdot 7$ & 11.9 & $13.7(6)$ & $15 \cdot 5(6)$ & $12.9(5)$ & $12.9(6)$ & $14 \cdot 0(7)$ & $17 \cdot 0(6)$ & $17 \cdot 5(6)$ & 187 & 355 \\
\hline Appendicectomy $(440-5)$ & $29 \cdot 6(3)$ & $35 \cdot 1(2)$ & $37 \cdot 2(2)$ & $34.9(2)$ & $33 \cdot 4(2)$ & $26 \cdot 0(2)$ & $24 \cdot 3(2)$ & $23.8(2)$ & $24 \cdot 2(2)$ & $20 \cdot 1(3)$ & 323 & 492 \\
\hline Orchidopexy (648) & $24 \cdot 1(5)$ & $19 \cdot 1(6)$ & $12 \cdot 6(8)$ & $10 \cdot 8(9)$ & $9 \cdot 8$ & $6 \cdot 8$ & $4 \cdot 7$ & $2 \cdot 1$ & 3.0 & $3 \cdot 0$ & 34 & 297 \\
\hline Circumcision $(660-1)$ & $22 \cdot 9(6=)$ & $19 \cdot 7(5)$ & $14 \cdot 1(6)$ & 8.9 & $5 \cdot 8$ & $3 \cdot 3$ & $3 \cdot 1$ & $4 \cdot 1$ & $6 \cdot 4$ & $8 \cdot 6(9)$ & 50 & 282 \\
\hline Operations on nails $(927-8)$ & $1 \cdot 3$ & 3.0 & $5 \cdot 1$ & $11 \cdot 0(8)$ & $20 \cdot 3(4)$ & $19 \cdot 6(4)$ & $17 \cdot 7(4)$ & $16 \cdot 4(5)$ & $16 \cdot 1(7)$ & $12 \cdot 6(7)$ & 16 & 311 \\
\hline
\end{tabular}

$\star \mathrm{ICD}=$ International Classification of Diseases (WHO); OPCS $=$ Office of Population Censuses and Surveys.

$f n_{1}$ and $n_{2}$ are the numbers of people on which the highest and lowest rates for each clinical disorder are based.

CLINICAL DISORDERS: MALES

Table 2 shows age specific admission rates for individual disorders. Disorders were chosen for inclusion if they were in the 10 most common disorders in at least one year of age. Head injury was the most common reason for hospital admission in males throughout the age range, admission rates increasing slightly in older adolescence. In boys aged 10 years admission for operations on tonsils and adenoids was the second most common reason for hospital admission followed by appendicectomy. By 14 years of age, head injury remained the most common reason for hospital admission, followed by appendicectomy and operations on the nose (notably for fracture of the nose), which was double the rate for girls throughout the age range. By the age of 19 years, head injury remained the most common reason for hospital admission, followed by open wounds and appendicectomy.

Admission rates which decreased substantially with age included those for respiratory disorders, particularly asthma, which decreased more with age in males than in females, abdominal symptoms (without appendicectomy), contrasting with increasing rates in females, fractured radius and ulna, myringotomy, operations on tonsils and adenoids, orchidopexy and circumcision, though the latter increased again between ages 16 and 19 years. Appendicectomy decreased from the age of 12 years. Disorders that increased with age included open wounds, poisoning (mainly deliberate self poisoning ${ }^{5}$ ), operations on nose, operations on teeth (notably dental extraction), and operations on nails (notably ingrowing toenails).

\section{CLINICAL DISORDERS: FEMALES}

In girls aged 10 years, the most common reasons for general hospital admission were operations on tonsils and adenoids, appendicectomy, head injury and myringotomy (table 3). By the age of 15 years the most common reason for hospital admission was ter- mination of pregnancy. The most common reasons for hospital admission at $\mathbf{1 5}$ years were operations on tonsils and adenoids, appendicectomy, and poisoning. Admission rates for termination of pregnancy increased sharply from 15 to 16 years of age. The most common reasons for hospital admission of girls from 16 years were for obstetric care and for termination of pregnancy. By the age of 19 years, the next most common reasons, after these, for hospital admission were operations on teeth, abdominal symptoms (without appendicectomy), postnatal operations, and poisoning.

Admission rates decreased substantially with age for respiratory disorders, fractured radius and ulna (the rate in females was a quarter of that for males by age 19 years), myringotomy, and operations on tonsils and adenoids. Tonsillectomy none the less remained common in older teenage females, considerably more so than in males. Admission rates increased with age for disorders associated with pregnancy, for dilatation and curettage, poisoning, and operations on teeth. For these latter two disorders the rate for females was more than double the rate for males by the age of 19 years. Admission rates for abdominal symptoms (without appendicectomy) increased with age and contrasted with admission rates for males in this respect. Admission rates for appendicectomy increased and decreased with age, peaking at ages 14-16 years and thereafter decreasing through adult life.

\section{Discussion}

Detailed disease profiles through adolescence are not available from routine statistics in England, though it is known that hospital admission rates are lower at ages 10-14 and 15-19 years than at most other five year age groups. ${ }^{6}$ As judged from the published (unlinked) data on the grouped ages 10-14 and 15-19 years, the general profile by diagnosis and operation is similar in this study to that in England as a whole, with the exception that admission rates for fractured radius and 
Table 3 Hospital admission rates in females expressed as rates per 10000 females at each age, and the range of numbers on which the rates are based, with the top 10 reasons for admission (excluding obstetrics) at each age ranked in parentheses

\begin{tabular}{|c|c|c|c|c|c|c|c|c|c|c|c|c|}
\hline \multirow{2}{*}{$\begin{array}{l}\text { Diagnoses (and ICD codes) or } \\
\text { operations (and OPCS } \\
\text { codes in italics) }\end{array}$} & \multicolumn{10}{|l|}{ Age (years) } & \multicolumn{2}{|c|}{ Range† } \\
\hline & 10 & 11 & 12 & 13 & 14 & 15 & 16 & 17 & 18 & 19 & $n_{1}$ & $n_{2}$ \\
\hline $\begin{array}{l}\text { Upper respiratory tract } \\
\text { infections }(460-5,478) \\
\text { Asthma }(493)\end{array}$ & $\begin{array}{l}9 \cdot 9(7=) \\
9 \cdot 9(7=)\end{array}$ & $\begin{array}{l}7 \cdot 9(10) \\
8 \cdot 6(8)\end{array}$ & $\begin{array}{l}7 \cdot 7(8) \\
5 \cdot 8(10)\end{array}$ & $\begin{array}{l}8 \cdot 2(8=) \\
6 \cdot 7(10)\end{array}$ & $\begin{array}{l}7 \cdot 8(9) \\
6 \cdot 5\end{array}$ & $\begin{array}{l}6 \cdot 7(10) \\
5 \cdot 4\end{array}$ & $\begin{array}{l}6 \cdot 4 \\
4 \cdot 1\end{array}$ & $\begin{array}{l}7 \cdot 6 \\
5 \cdot 5\end{array}$ & $\begin{array}{l}6 \cdot 1 \\
7 \cdot 1\end{array}$ & $\begin{array}{l}5 \cdot 2 \\
7 \cdot 1\end{array}$ & $\begin{array}{l}74 \\
58\end{array}$ & $\begin{array}{l}115 \\
115\end{array}$ \\
\hline $\begin{array}{l}\text { Inflammatory disease of ovary, } \\
\text { fallopian tubes }(614) \\
\text { Abdominal symptoms }(789) \\
\text { Fractured radius, ulna (813) }\end{array}$ & $\begin{array}{l}0 \cdot 0 \\
21 \cdot 7(5) \\
13 \cdot 5(6)\end{array}$ & $\begin{array}{l}0 \cdot 1 \\
19 \cdot 6(4) \\
10 \cdot 5(7)\end{array}$ & $\begin{array}{r}0.4 \\
18 \cdot 7(5) \\
6 \cdot 8(9)\end{array}$ & $\begin{array}{c}0 \cdot 3 \\
18 \cdot 2(5) \\
4 \cdot 3\end{array}$ & $\begin{array}{c}0 \cdot 7 \\
21 \cdot 8(6) \\
2 \cdot 3\end{array}$ & $\begin{array}{c}1 \cdot 6 \\
25 \cdot 2(6) \\
0.9\end{array}$ & $\begin{array}{c}6 \cdot 0 \\
30 \cdot 9(5) \\
0 \cdot 8\end{array}$ & $\begin{array}{l}8 \cdot 6(10) \\
36 \cdot 4(3=) \\
1 \cdot 1\end{array}$ & $\begin{array}{l}13.6(9) \\
40.5(3) \\
1.1\end{array}$ & $\begin{array}{l}16 \cdot 3(10) \\
44 \cdot 1(3) \\
0 \cdot 8\end{array}$ & $\begin{array}{r}0 \\
234 \\
11\end{array}$ & $\begin{array}{l}233 \\
630 \\
157\end{array}$ \\
\hline $\begin{array}{l}\text { Head injury, concussion } \\
\quad(850,854) \\
\text { Poisoning (960-989) } \\
\text { Myringotomy (193) }\end{array}$ & $\begin{array}{l}22 \cdot 8(3) \\
1: 8 \\
22 \cdot 4(4)\end{array}$ & $\begin{array}{c}26 \cdot 1(3) \\
2 \cdot 1 \\
15 \cdot 1(5)\end{array}$ & $\begin{array}{c}24 \cdot 3(3) \\
3 \cdot 4 \\
10 \cdot 6(6)\end{array}$ & $\begin{array}{l}25 \cdot 1(3) \\
10 \cdot 3(6) \\
8 \cdot 2(8=)\end{array}$ & $\begin{array}{c}26 \cdot 3(3) \\
23 \cdot 6(5) \\
6 \cdot 0\end{array}$ & $\begin{array}{l}25 \cdot 7(5) \\
31 \cdot 6(4) \\
3 \cdot 8\end{array}$ & $\begin{array}{l}27 \cdot 0(6) \\
34 \cdot 5(4) \\
2 \cdot 6\end{array}$ & $\begin{array}{l}24 \cdot 4(7) \\
38 \cdot 5(2) \\
1 \cdot 9\end{array}$ & $\begin{array}{l}28 \cdot 3(7) \\
39 \cdot 0(4) \\
1 \cdot 7\end{array}$ & $\begin{array}{l}23 \cdot 0(8) \\
37 \cdot 5(5) \\
1 \cdot 2\end{array}$ & $\begin{array}{r}265 \\
21 \\
17\end{array}$ & $\begin{array}{l}404 \\
558 \\
260\end{array}$ \\
\hline $\begin{array}{l}\text { Operations on nose } \\
(210-1,215-24) \\
\text { Tonsils and adenoids }(230-9) \\
\text { Operations on teeth }(250-9) \\
\text { Appendicectomy }(440-5) \\
\text { Dilatation and curettage }\end{array}$ & $\begin{array}{c}6 \cdot 0(10) \\
54 \cdot 2(1) \\
8 \cdot 3(9) \\
25 \cdot 7(2)\end{array}$ & $\begin{array}{r}8 \cdot 5(9) \\
42 \cdot 1(1) \\
13 \cdot 2(6) \\
26 \cdot 6(2)\end{array}$ & $\begin{array}{r}9 \cdot 0(7) \\
40 \cdot 9(1) \\
19 \cdot 3(4) \\
25 \cdot 8(2)\end{array}$ & $\begin{array}{l}10 \cdot 0(7) \\
36 \cdot 1(1) \\
23 \cdot 8(4) \\
32 \cdot 3(2)\end{array}$ & $\begin{array}{l}10 \cdot 0(8) \\
34 \cdot 4(1) \\
24 \cdot 0(4) \\
34 \cdot 2(2)\end{array}$ & $\begin{array}{l}9 \cdot 8(8) \\
44 \cdot 3(2) \\
22 \cdot 3(7) \\
36 \cdot 3(3)\end{array}$ & $\begin{array}{r}9 \cdot 4(9) \\
36 \cdot 9(2) \\
26 \cdot 6(7) \\
34 \cdot 9(3)\end{array}$ & $\begin{array}{l}8 \cdot 2 \\
36 \cdot 4(3=) \\
31 \cdot 6(5) \\
31 \cdot 5(6)\end{array}$ & $\begin{array}{l}8 \cdot 6 \\
35 \cdot 5(5) \\
43 \cdot 7(2) \\
31 \cdot 9(6)\end{array}$ & $\begin{array}{l}7 \cdot 2 \\
30 \cdot 2(7) \\
48 \cdot 8(2) \\
31 \cdot 7(6)\end{array}$ & $\begin{array}{r}70 \\
431 \\
171 \\
299\end{array}$ & $\begin{array}{l}137 \\
630 \\
874 \\
507\end{array}$ \\
\hline $\begin{array}{l}\text { Dilatation and curettage } \\
(703-4)\end{array}$ & $0 \cdot 0$ & $0 \cdot 0$ & 0.3 & $0 \cdot 7$ & 1.6 & $2 \cdot 3$ & $4 \cdot 5$ & $7 \cdot 0$ & $12 \cdot 1(10)$ & $17 \cdot 9(9)$ & 4 & 256 \\
\hline $\begin{array}{l}\text { Termination of pregnancy } \\
\text { (742) } \\
\text { Postnatal operations }(770-9)\end{array}$ & $\begin{array}{l}0.0 \\
0.0\end{array}$ & $\begin{array}{l}0 \cdot 0 \\
0 \cdot 1\end{array}$ & $\begin{array}{l}0 \cdot 3 \\
0 \cdot 1\end{array}$ & $\begin{array}{l}2 \cdot 5 \\
0 \cdot 2\end{array}$ & $\begin{array}{c}16 \cdot 5(7) \\
1.7\end{array}$ & $\begin{array}{c}48 \cdot 3(1) \\
3 \cdot 4\end{array}$ & $\begin{array}{l}79 \cdot 6(1) \\
11 \cdot 2(8)\end{array}$ & $\begin{array}{l}85 \cdot 4(1) \\
21 \cdot 0(8)\end{array}$ & $\begin{array}{l}83 \cdot 7(1) \\
28 \cdot 0(8)\end{array}$ & $\begin{array}{l}82 \cdot 4(1) \\
41 \cdot 1(4)\end{array}$ & $\begin{array}{l}4 \\
1\end{array}$ & $\begin{array}{r}1218 \\
587\end{array}$ \\
\hline $\begin{array}{l}\text { Operations on joints }(801,803 \text {, } \\
806,815,816,821-828)\end{array}$ & $2 \cdot 2$ & $2 \cdot 6$ & $3 \cdot 3$ & $4 \cdot 6$ & $7 \cdot 2(10)$ & $7 \cdot 7(9)$ & $8 \cdot 0(10)$ & $10 \cdot 2(9)$ & $9 \cdot 9$ & $10 \cdot 0$ & 26 & 143 \\
\hline
\end{tabular}

$\star \mathrm{ICD}=$ International Classification of Diseases (WHO); OPCS = Office of Population Censuses and Surveys.

$f n_{1}$ and $n_{2}$ are the numbers of people on which the highest and lowest rates for each clinical disorder are based.

ulna are higher in England than in Oxford. ${ }^{6}$ This may reflect geographical variation in the threshold for admission for such fractures.

Studies of adolescent health, notably from the USA, report that it is affected in particular by risk taking behaviour resulting in road traffic accidents, other violence, substance abuse, and, for girls, unwanted pregnancies. ${ }^{78}$ The single most common reason for hospital admission in males in our study was head injury, with an average annual admission rate ranging from 46-67/10 000 males. This compares with a range of $23-28 / 10000$ in females and no doubt reflects differences in behaviour patterns and risk taking activities. These rates are slightly higher than those found in studies of head injury carried out in the USA, which reported admission rates of between 15-20/10 $000 .{ }^{9}$ This may reflect differences in criteria for hospital admission or perhaps differences in the definition of head injury. The age related increase in admissions for head injury and the gender differences found in this study are similar to those reported elsewhere. ${ }^{9-11}$ Other injuries such as fractures probably occur much more often than head injuries but can be dealt with safely without admission, whereas patients presenting with head injuries are often admitted for observation. ${ }^{10}$

The most common reason for hospital admission of older adolescent girls was to give birth. The next most common reason was termination of pregnancy. The data in this study represent only those terminations carried out in National Health Service hospitals and are therefore a considerable underestimate. Approximately the same number of terminations of pregnancy in this age group in this region are carried out privately. ${ }^{12}$ The Oxford region is fairly representative nationally in discharge rates for termination of pregnancy. ${ }^{12}$ Total numbers have risen steadily and one third are in women under the age of $20 .^{13}$ The peak of terminations in the teenage years may be influenced by cohort effects as well as chronological age. Conception under the age of 16 years is one of the key areas in The Health of the Nation. ${ }^{14}$ The target is to reduce the rate of conception in this age group by $50 \%$ by the year 2000. The target set by the Faculty of Public Health Medicine is 10/1000 15 year olds and none in girls under 15 by the year 2000. ${ }^{15}$

Care in the surgical specialties was considerably more common throughout the age range than that in paediatrics and general medicine. For example, appendicectomy was common throughout adolescence in males and females, peaking in girls at age 15 and in boys at age 12 years. The admission rates, $20-37 / 10000$, are similar to those reported by Addiss et al in the USA, ${ }^{16}$ except that they reported higher admission rates for males at all ages (grouped 10-14 and 15-19 years).

Care under the designation of paediatrics accounted for only a minority of all care of 10 and 11 year olds and for a small minority of care by the age of 14 and 15 years. The shift in care from paediatrics to general medicine at about 14 years is illustrated in table 1 and reflects clinical policy on the age divide between the specialties. The National Association for the Welfare of Children in Hospital (now Action for Sick Children) recommends that adolescents are accommodated in a separate and self contained area linked to the paediatric department and that their care under the specialist should also be under the general oversight of the consultant paediatrician. ${ }^{2}$ The Audit Commission has also highlighted the special needs of adolescents, in particular with regard to the ward environment. ${ }^{17} \mathrm{~A}$ disturbing hospital episode in the teenage years (for example, being placed next to a very ill adult) may leave lasting impressions, affecting attitudes to medical care that may persist throughout adult life. Purchasers and providers of health care will wish to ensure that adolescents are treated in the most suitable ward space available for 
them. Local information on profiles of care by specialty and ward in this age group are therefore important. ${ }^{2}$

In summary, most admissions in this age group are for surgical rather than medical disorders. Some of the most common reasons for admission, such as injury, self poisoning, and unwanted pregnancy, are behavioural in origin and potentially preventable. The scope for prevention and for ensuring the wellbeing of teenagers starts in childhood.

The Unit of Health-Care Epidemiology is part of the Department of Public Health and Primary Care, University of Oxford. The unit is funded by the Department of Health and also receives support from the Oxford Regional Health Authority. We thank Pamela Evans for secretarial help.

1 Bewley BR, Walsworth-Bell J. The inadequacy of adolescent health statistics. Community Medicine 1982; 4: 97-9. 2 National Association for the Welfare of Children in Hospital. Setting standards for adolescents in hospital. London: NAWCH, 1990.

3 World Health Organisation. International classification of diseases. 9th Revision. Geneva: WHO, 1977.

4 Office of Population Censuses and Surveys. Classification of surgical operations. 3rd Revision. London: OPCS, 1975.

5 Sellar C, Goldacre MJ, Hawton K. Reliability of routine hospital data on poisoning as measures of deliberate selfpoisoning. F Epidemiol Community Health 1990; 44: 313-5.
6 Department of Health and Social Security; Office of Population Censuses and Surveys. Hospital in-patient enquiry: summary tables, 1985. London: HMSO, 1987. (Series MB4 No 26.)

7 Blum R. Contemporary threats to adolescent health in the United States. $\mathfrak{F} A M A$ 1987; 257: 3390-5.

8 McManus M, McCarthy E, Kozak LJ, Newacheck P. Hospital use by adolescents and young adults. fournal of Adolescent Health 1991; 12: 107-15.

9 MacKenzie EJ, Edelstein SL, Flynn JP. Trends in hospitalized discharge rates for head injury in Maryland, hospitalized discharge rates for head injury in

10 Brookes M, MacMillan R, Cully S, et al. Head injuries in accident and emergency departments. How different are children from adults? F Epidemiol Community Health 1990; 44: $147-51$

11 Logan RFL, Ashley JSA. Health problems of adolescents and young adults. In: Hobson W, ed. The theory and practice of public health. 3rd Ed. London: Oxford University Press, 1969: 352-65.

12 Office of Population Censuses and Surveys. Abortion statistics. London: HMSO, published annually. (Series 4B.)

13 Pearson JF. Preventing unwanted pregnancies. BMF 1991; 303: 598 .

14 Secretary of State for Health. The health of the nation: a strategy for health in England. London: HMSO, 1992. (Cmnd 1986.)

15 The Faculty of Public Health Medicine of the Royal Colleges of Physicians. UK levels of health. First report. London: The Faculty of Public Health Medicine, 1991.

16 Addiss DG, Shaffer N, Fowler BS, Tanxe RV. The epidemiology of appendicitis and appendicectomy in the United States. Am 7 Epidemiol 1990; 132: 910-25.

17 Audit Commission. Children first. A study of hospital services. London: HMSO, 1993: 31-2. 